\title{
Contextos de crianza del niño. Problemas y prospectiva*
}

\section{Urie Bronfenbrenner **}

\author{
Universidad de Cornell
}

Traducción: Amelia Alvarez

Este artículo se basa, fundamentalmente, en datos ausentes. La capacidad de la psicología, tanto en calidad de disciplina como de profesión, para hacer frente a los problemas que surgen en los contextos de crianza del niño topa con un vacío en el conocimiento científico. Nuestra ciencia es particularmente unidireccional. Sabemos mucho más de los niños que de los entornos en los que viven o de los procesos mediante los cuales esos entornos afectan al curso del desarrollo. De ahi que nuestra capacidad para tomar la palabra en asuntos de política social sobre todo lo referente a los contextos en que el niño crece se vea consecuentemente limitada. Nosotros creemos que este hecho aumenta nuestra obligación de equilibrar la balanza en lugar de disminuirla.

\section{EL CONTEXTO AUSENTE}

La escasez de investigaciones y de conocimientos apropiados sobre los contextos del desarrollo humano es, en una importante medida, el fruto de la ausencia de un marco teórico pertinente para analizar los entornos en los que los seres humanos viven. Los conceptos existentes se limitan a unas pocas categorías en bruto e indiferenciadas que hacen poco más que localizar a la gente en términos de su "locación» social: el escenario del que proceden. Así, un examen de los estudios de las influencias ambientales sobre el desarrollo revela las siguientes tipologías morales para describir contextos de conducta y crecimiento: tamaño de la familia, posición ordinal, un solo padre versus ambos padres al frente del hogar, madre trabajando versus madre no trabajando, cuidado del niño en casa versus cuidado fuera de casa, padres versus

\footnotetext{
* "Contexts of Child Rearing Problems and Prospects", American Psycbologist, 1979, 34 (10), 844-8 90. Reproducido con autorización. ( De esta traducción, Infancia y Aprendizaje, 1985.

** Dirección del autor: Department of Human Development and Family Studies, Cornell University, Martha Van Rensselaer Hall, Ithaca, Nueva York 148, 3. Estados Unidos.
} 
iguales $\mathrm{y}$, lo que quizá sea lo. más frecuente, clase social u origenes étnicos. Por otra parte, y en una abrumadora proporción, los datos de esos estudios consisten en información, no sobre los escenarios de los que proceden las personas, sino sobre las características de las propias personas, esto es, cómo difieren entre sí personas provenientes de diferentes contextos.

Yo sostengo que ese tipo de comparaciones: que se centran exclusivamente en las consecuencias no representan en absoluto una estrategia a elegir en la investigación sobre el desarrollo humano. En concreto, añaden muy poco a nuestra comprensión de cómo afectan los contextos ecológicos al curso del desarrollo psicológico. Por ejemplo, algunos factores del entorno - como estatus ocupacional, cantidad de escolarización o, en último término, tamaño de la familia - parecen tener una sustancial y duradera influencia sobre los resultados del desarrollo. Otros - como el de familia de padre único versus familia de dos padres, o contacto continuado con adultos versus contacto con iguales - parecen producir efectos significativos que varian sistemáticamente según lugar y tiempo. Por último, otros - como empleo materno o cuidado del niño en guardería- muestran, según las mediciones actuales, huellas inconsistentes, efímeras y de pequeña magnitud.

¿Qué es lo que explica esas variaciones en el impacto de los diferentes tipos de entornos humanos? Dadas las características de los diseños utilizados en la investigación al uso, la pregunta no puede ser contestada, puesto que ni las variables independientes ni las dependientes utilizadas habitualmente pueden aportar información alguna sobre los procesos implicados. Las primeras consisten en tipologías ambientales basadas en lo que Lewin (1935) llamaba fenotipos y genotipos: rasgos externos, específicos, de la situación más que propiedades transculturales generalizables a una amplia variedad de contextos. En cuanto a los resultados evolutivos, se miden normalmente sin hacer referencia a las propiedades del escenario físico y social en el que se efectúa la evaluación o al significado de la conducta observada en un escenario o en otro. En suma, todavia carecemos de una taxonomía específica tanto para analizar escenarios en términos de parámetros evolutivamente relevantes como para evaluar el desarrollo en términos de resultados ambientalmente relevantes.

Afortunadamente, hay excepciones a la regla, y en la psicología evolutiva dominante la más notable es el sustancial cuerpo de investigación avalando la primacía de los procesos interpersonales en la evolución de la conducta. Me estoy refiriendo a mecanismos tan familiares como refuerzo, modelado, identificación y aprendizaje social. La naturaleza de estos fenómenos establecidos refleja tanto la consistencia como las limitaciones de nuestro actual conocimiento. La consistencia estriba en el demostrado poder de esos procesos para modelar la conducta y el desarrollo. Las limitaciones son de tres tipos. En primer lugar, y a un nivel puramente descriptivo, contamos con poca información acerca de cómo se distribuyen dichos procesos en los diferentes contextos de crianza y desarrollo del niño. En segundo lugar, y desde un punto de vista más analítico, nos faltan conocimientos sobre las condiciones ecológicas que soportan, estimulan o entorpecen la actuación de esos procesos. En tercer lugar, todos esos procesos son 
esencialmente diádicos, es decir, están limitados al intercambio entre personas consideradas en situaciones duales.

La principal tesis de este artículo es que estamos en condiciones actualmente de superar esas limitaciones mediante una síntesis teórica del conocimiento existente sobre los contextos interpersonales, por un lado, y de los conceptos y datos que están emergiendo últimamente y que apuntan a unos parámetros transcontextuales de entornos de crianza más allá del nivel diádico, por otro; sostendré, además, que esta integración teórica permite extraer patrones consistentes de los resultados de investigaciones de que disponemos y que son importantes tanto para el conocimiento científico como para la política social relacionada con los contextos de crianza del niño en las modernas sociedades industrializadas.

Es mi opinión que el actual conocimiento acerca de la socialización nos permite especificar cuatro propiedades del entorno ecológico que favorecen los procesos del desarrollo humano. Las propuestas I $_{\text {y }} 2$ presentadas más adelante, enuncian dos tipos de condiciones complementarias que deben darse en los escenarios (settings) para que el desarrollo tenga lugar. La propuesta 3 define el papel crítico de los terceros (tanto intra como interescenarios) como soportes y estimuladores de los sistemas evolutivos. Finalmente, la propuesta 4 estipula determinadas formas de interconexión entre los escenarios que facilitan el funcionamiento interno de esos escenarios como contextos para el desarrollo humano.

\section{CONTEXTOS DE DESARROLLO PRIMARIOS Y SECUNDARIOS}

Estos dos primeros tipos de condiciones necesarias son mutuamente excluyentes en el tiempo, pero pueden tener lugar secuencialmente bien dentro del mismo escenario o de un escenario al siguiente. La propuesta I es un prerrequisito para la propuesta 2.

Propuesta $x$. Un contexto de desarrollo primario es aquel en el que el niño puede observar e incorporarse a patrones en uso de actividad progresivamente más compleja, conjuntamente o bajo la guía directa de personas que poseen conocimientos o destrezas todavía no adquiridas por el niño y con las cuales éste ha establecido una relación emocional positiva.

Propuesta 2. Un contexto de desarrollo secundario es aquel en el cual se ofrecen al niño oportunidades, recursos y estímulos para implicarse en las actividades que ha aprendido en los contextos de desarrollo primarios, pero ahora sin la intervención activa o la guia directa de otra persona poseedora del conocimiento o destreza que supere a la del niño.

Es claro que la propuesta 2 no puede ser operativa sin la experiencia estipulada en la propuesta I. Pero lo inverso es igualmente cierto: si no hay escenarios en los que el niño pueda ejercitar las capacidades recién adquiridas sin la intervención de otras personas más competentes, el proceso de desarrollo será incompleto.

Tal como se han enunciado, estas dos propuestas pueden parecer poco más que pedantes formulaciones del sentido común, pero de hecho han sido extraídas de un importante volumen de investigaciones revisadas en otro lugar (Bronfenbrenner, 1979). La mayor parte de las 
investigaciones pertinentes han sido llevadas a cabo con familias, tanto en el laboratorio como en el hogar. Sin embargo, en los últimos años, las investigaciones dirigidas al estudio de los procesos han tenido en cuenta también otros escenarios, incluyendo habitaciones de clínicas de maternidad (Scarr-Salapatek y Williams, 1973), instituciones para niños (Tizard y Hodges, 1978), guarderías de día y hogares-guardería (Belsky y Steinberg, 1978; Cochran, 1977; Ruopp, Travers, Glantz y Coelen, 1979); centros de preescolar (Bronfenbrenner, 1974a; Lazar, Hubbell, Murray, Rosche y Royce, I977); aulas escolares (Smith, 1968; Hayes y Grether, nota I); campamentos (Sherif, Harvey, Hoyt, Hood y Sherif, 196I) y comunidades completas (Ogbu, I 974).

Una comparación de los resultados de estas investigaciones revela convincentes y sólidas evidencias que respaldan los criterios estipulados en la proposición I y 2 como condiciones necesarias para el efectivo funcionamiento de los entornos inmediatos en tanto que contextos de desarrollo humano. Por otra parte, como se demuestra en algunos de los estudios citados, los criterios pueden ser operacionalizados y trazado su impacto sobre las consecuencias evolutivas de modo que sea de hecho posible hacer una evaluación de los procesos y sus efectos. Por citar sólo un ejemplo, Hales, Lozoff, Sosa y Kennell (1977), confirmaron experimentalmente la existencia de un periodo crítico de doce horas posteriores al nacimiento en las que la oportunidad de una interacción continuada entre la madre y el recién nacido en el hospital deriva en cambios evolutivos en la conducta tanto de la madre como del niño, cambios que otros investigadores de la Western Reserve demostraron poder detectar todavia cuando los niños tenían cinco años (Kennell, Trause y Klaus, 1975); Ringler, nota 2.

El significado de las propuestas I y 2 sobrepasa el dominio del conocimiento científico hacia lo que yo considero acuciantes demandas de política social. Puede vaticinarse que si examináramos sistemáticamente los contextos reales en los que los niños de nuestra sociedad pasan sus horas de vigilia, nos encontraríamos con que muchos de los escenarios adolecen en gran medida de ambos conjuntos de requisitos. Precisando más, en muchos lugares y durante muchas horas, es muy probable que los niños no tengan a su alcance adultos capacitados que los impliquen en actividades conjuntas progresivamente más complejas, ni posiblemente el tipo de situación propicie recursos e incentivos para que el niño se entregue a complejas actividades previamente aprendidas.

Me apresuro a señalar, para aquellos lectores que se convencen demasiado fácilmente o para aquellos que prefieren mantener un sano escepticismo, que no hay datos que permitan probar mi pesimista predicción. Por razones que ya he indicado, los investigadores no han efectuado análisis sistemáticos, basados en muestras representativas, sobre los entornos en los que viven cotidianamente los niños y las personas que están a su cargo. Hay alguna evidencia de que es más probable que los requisitos enunciados en las propuestas i y 2 falten en las instituciones para niños (Bronfenbrenner, 1979, págs. 1 32-163) y en los hogares de acogida (Fanshel y Shinn, 1978; Cruber, 1978). Pero sólo el 4 por roo de los niños estadounidenses están actualmente en tales instituciones, mientras que mi predicción es aplicable a la gran mayoría. Por añadidura, está basada únicamente en evidencias indirectas y en una cadena de inferencias que requiere críticas, desafios $y$, 
sobre todo, contrastación empírica. Desde un punto de vista científico, la predicción resulta, por consiguiente, más apropiada si se formula así: ¿Hasta qué punto los profundos y rápidos cambios que han tenido lugar en la familia americana en las tres últimas décadas, y especialmente desde 1970 (Bronfenbrenner, 1975; Toward a National Policy for Children and Families, 1976) han hecho dificil a los padres el procurar las condiciones de desarrollo estipuladas en las propuestas i y 2 ? Un análisis retrospectivo está fuera de lugar, puesto que no existen datos sobre ninguno de los cambios en el funcionamiento de la familia actual ni en la conducta de los niños durante ese período. Siempre habrá, por supuesto, la posibilidad de emprender estudios prospectivos basados en la no insensata esperanza de que las tendencias actuales no se van a invertir, pero pasaría algún tiempo antes de que pudieran recogerse datos que reflejen efectos del cambio. Sí podrían obtenerse resultados pertinentes con mucha mayor rapidez a través de estudios transversales que contrasten entornos familiares.

Sin embargo, cualquier esfuerzo científico en este sentido, tropezará con la ausencia de una perspectiva teórica lo suficientemente amplia y diferenciada como para investigar el desarrollo en su contexto. El omnipresente uso de un modelo diádico padres-hijo excluye considerar la posibilidad de que fuerzas externas al sistema bipersonal pudieran estar influyendo en su eficacia y resultados. Las fuerzas más próximas de este tipo emanan de las terceras partes presentes en el escenario inmediato.

\section{EL IMPACTO DE LAS TERCERAS PARTES EN. EL ESCENARIO}

Partiendo del hecho de que la familia nuclear es un sistema tripersonal, resulta sorprendente que tan reducido número de investigadores hayan centrado su interés en esta propiedad estructural distintiva. Incluso el reciente caudal de investigaciones sobre la influencia de los padres (fathers) sobre el desarrollo de sus hijos (Lamb, 1976) ha seguido manteniendo el paradigma diádico, en su mayor parte mediante la simple sustitución de la madre por el padre en el modelo analítico. Sin embargo, están comenzando a surgir algunos datos que reflejan la influencia de terceras partes en el funcionamiento del sistema padre (parent)-hijo. Parke (1978) observó a ambos padres con su recién nacido en el escenario del hospital para determinar qué efectos causaba cada uno de los padres en la interacción del otro con su hijo. Tanto para el padre como para la madre, la interacción con el niño aumentaba con la presencia del cónyuge. Dentro de una línea similar, Pederson (nota 3 ), en un estudio que combinaba la entrevista con técnicas observacionales, halló que cuando el padre constituia un apoyo para la madre, ésta era más efectiva en la alimentación de su hijo, mientras que el conflicto matrimonial iba asociado a una ineptitud por parte de la madre en dicha alimentación.

Estudios llevados a cabo con grupos de edades superiores respecto a las consecuencias del divorcio han obtenido resultados expuestos por los autores en este mismo número de American Psychologist (Hetherington, Cox y Cox, 1976, 1978). Sus datos documentaban la progresiva 
desorganización de la díada madre-hijo como sistema socializador consecuente a la desaparición del padre del hogar, así como perturbaciones concomitantes en la conducta manifiesta del niño. Más relevantes, sin embargo, para el tema que nos ocupa son las excepciones a la regla: niños del divorcio que no manifestaban muestras de un funcionamiento psicológico perturbado. Estos niños tendian a ser jóvenes cuyas madres «estaban disponibles, mantenían una disciplina firme, pero sensible y estimulaban una conducta independiente" (Hetherington, Cox y Cox, nota 4 , pág. ${ }_{3}$ ). Los investigadores se planteaban entonces la pregunta de qué factores podrían explicar la capacidad de esas madres para funcionar eficazmente respecto a sus hijos. La influencia más crítica en este sentido resultaba ser la conducta del padre. Si éste continuaba apoyando el papel de la madre, la relación de ésta con el niño era más armoniosa y el ajuste del niño se veía relativamente poco afectado. Inversamente, el conflicto sostenido entre los padres divorciados iba asociado con la perturbación en el funcionamiento de la diada madre-hijo y en la conducta del niño (Hetherington, et al., 1976, 1978).

Una influencia similar se observó en el resto de las terceras partes (abuelos, amigos y vecinos) pero ninguno de éstos eran tan cruciales como el padre divorciado que mantenía una relación de apoyo con su esposa.

$\mathrm{El}$ anteriormente mencionado conjunto de hallazgos conduce a una tercera propuesta que especifica una propiedad más de un contexto eficaz para la crianza del niño.

Propuesta 3. El desarrollo potencial de un escenario depende del grado en que las terceras partes presentes en el escenario apoyen o socaven las actividades de aquellos actualmente implicados en la interacción con el niño.

Aunque los resultados de su trabajo respalden claramente la propuesta 3 , Hetherington y sus colegas, en su intento de establecer el factor más importante que determina la conducta y desarrollo en las madres divorciadas, apuntaron a factores externos a la propia familia. A su modo de ver, elas perturbaciones en el desarrollo no parecen poder atribuirse principalmente a lä ausencia del padre, sino a las tensiones y faltas de apoyo que surgen en el funcionamiento de la nueva familia para la madre sola y sus hijos... Resulta crucial desarrollar políticas sociales y mecanismos creativos que reduzcan las tensiones y nuevos sistemas de apoyo para las familias de padre único (Hetherington, et al, nota 4, págs. 31-32).

\section{INTERRELACIONES ENTRE LOS ESCENARIOS}

La afirmación anterior nos lleva directamente a la consideración de una segunda área importante de investigación, imprescindible para un mejor entendimiento del contexto de crianza del niño: un examen de las relaciones entre los escenarios y su importancia para los procesos evolutivos. Voy a centrarme, en primer lugar, en las conexiones entre aquellos escenarios en los que el niño participa, como el hogar, la guardería, el preescolar y la escuela. A pesar del amplio número de investigaciones que documentan los logros evolutivos en cada uno de estos escenarios, solamente un reducido número ha tenido en cuenta el 
impacto de la experiencia del niño en un escenario sobre su conducta o desarrollo en otro (v. g. Elder, 1974; Elder y Rockwell, 1978; Scarr-Salapatek y Williams, 1973; Hayes y Grether, nota 1). Más raros todavia son los experimentos que busquen establecer relaciones entre los escenarios de cara a estimular la efectividad evolutiva de cada contexto.

Un ejemplo instructivo en este sentido lo proporciona Smith (1968). El objetivo de su experimento era mejorar los resultados escolares de las minorias de alumnos de clase baja en los grados elementales. El proyecto afectaba a unos r.000 niños, la mayoría de ellos de raza negra, provenientes de familias de bajo nivel de ingresos y que asistian a dos escuelas elementales públicas. Para adscribir a los padres en el programa, 30 madres voluntarias visitaron los hogares de su barrio en los que habia niños en edad de escolarización elemental. Entre otras actividades, se les pedia a los padres que oyeran leer a sus hijos, que les brindaran apoyo y elogios y les garantizaran un rato tranquilo para el estudio que no interfiriera con su programa de televisión favorito. A los niños de guarderia y preescolar se les daba libros para que se llevaran a casa y que llevaban la etiqueta «Por favor, léemelo». Se hizo un especial esfuerzo por implicar a los padres (fathers) en esta actividad y en que hicieran de narradores en la biblioteca. Se facilitó a los hogares un diccionario infantil y se animaba a las familias a poner su nombre en la cubierta interior. En reuniones periódicas, los padres planteaban sus problemas e ideas entre sí (por ejemplo, se ponian de acuerdo sobre el momento en el que a sus hijos se les pediria que estudiaran). A los maestros se les proporcionaba ayuda en las tareas de oficina por parte de estudiantes de escuelas empresariales que mecanografiaban y duplicaban los materiales necesarios. Un programa en marcha para maestros hacía hincapié en la influencia de los factores del entorno en la conducta de los niños en clase. Gente de color con empleos cualificados en la zona visitaban las clases y uhablaban de lo importante que habian sido las materias escolares para su vida posterior" (pág. I02).

La variedad e ingenuidad de las variables independientes en el experimento de Smith no se correspondian desgraciadamente con la elección de mediciones de resultados. En su forma cuantitativa, éstos se reducian a incrementos significativos en las ejecuciones en test de lectura y a las reacciones de los padres al programa expresadas a través de un cuestionario. El cuestionario aportó respuestas satisfactorias en un 90 por 100. Se expresaron actitudes especialmente favorables respecto al programa de estudio en el hogar y a las experiencias de lectura. Es de lamentar que no se obtuvieran datos comparativos de las conductas manifestadas por sujetos experimentales y de control en la clase o, igualmente importante, en la familia o en el grupo de amigos del barrio.

A pesar de estas limitaciones, el experimento de Smith ilustra otro principio que hace referencia a las condiciones que sustentan y estimulan la eficacia de los contextos específicos de crianza del niño.

Propuesta 4. El potencial de desarrollo de un escenario de crianza se ve incrementado en función del número de vínculos sustentadores entre ese escenario y otros contextos en los que se insertan el niño y los adultos responsables de su cuidado. Tales interrelaciones pueden 
adoptar la forma de actividades compartidas, comunicación en los dos sentidos e información suministrada a cada escenario sobre los demás.

Además de aportar ejemplos concretos de este principio, el experimento de Smith afronta un problema extendido que afecta a los niños en nuestra sociedad contemporánea occidental.

\section{LA ESCUELA COMO ESCENARIO ALIENADO}

Como ya he indicado en otros lugares (Bronfenbrenner, 1970, I974b, 1978), la escuela se ha ido aislando progresivamente del hogar, con importantes consecuencias para la conducta y el desarrollo de los niños. Mientras las escuelas de barrio desaparecen, los edificios escolares están más lejos, son más grandes y más impersonales. También las plantillas de personal aumentan en número, se planifican para una zona más amplia y viajan a - no residen en- la comunidad local. En consecuencia, hay menos posibilidades de que padres y maestros se conozcan, emprendan actividades conjuntas o se hagan amigos.

Por otra parte, el aislamiento no se da solamente entre la escuela y el hogar. En la medida en que las escuelas se trasladan a las afueras de las poblaciones se convierten en conglomerados física y socialmente aislados de la vida de la comunidad a la par que de la vida para la que supuestamente están preparando a los niños. El aislamiento se repite dentro de la propia escuela, donde se segrega a los niños en clases que, a menudo, cambian anualmente de configuración, tienen poca o ninguna identidad social por sí mismas y poca conexión entre sí o con la escuela como comunidad de todos; en la que sus 'miembros comparten una actividad responsable. Esta ausencia comparativa de vida común es caldo de cultivo para las fuerzas disruptivas, fruto de la segregación por edades que se da desde el momento en que los niños se ven apiñados con sus iguales. Es significativo el hecho de que los únicos adultos a los que se les pide que entren en este mundo de niños son personas con graduaciones de licenciatura con antecedentes muy similares y que reflejan muy poco la rica diversidad de la experiencia del mundo exterior.

Como resultado de todas estas fuerzas convergentes, la escuela se ha convertido a lo largo de las últimas dos décadas en uno de los más potentes focos de alienación de la sociedad americana. A mi modo de ver, es esa alienación la que subyace en el progresivo declive de las calificaciones que se han reflejado en los últimos doce años tanto en la población universitaria como en la población general de estudiantes de la educación primaria y secundaria (Harnischfeger y Wiley, nota s). Sus manifestaciones más dramáticas pueden obervarse en las crecientes tasas de homicidios, suicidios y delicuencia en los niños de edad escolar (Bronfenbrenner, 1975).

El barómetro de estas tendencias destructivas lo constituye el creciente nivel de vandalismo y violencia en las escuelas. Un informe del Comité del Senado de los Estados Unidos para asuntos judiciales (1975) resumía sus principales averiguaciones así: "Our Nation's Schools: A report Card " $A$ " in School Violence and Vandalism". El informe insistía en que el modelo no se limitaba a las grandes ciudades 
y sus zonas de marginación: se ha convertido en un fenómeno a escala nacional. No hay escuelas sin un presupuesto destinado a seguridad y, a menudo, a fuerzas del orden. Literalmente, la mano está ya escribiendo en la pared *.

El mensaje, para ser exactos, no ha sido dictado por datos rigurosos. A lo sumo, constituye una especulación fruto de la convergencia entre una teoria incompleta y unos hechos incompletos: como tal, tiene poca validez empírica. Pero creo que cumple una función social y científica de utilidad como hipótesis para estimular y orientar un trabajo empirico riguroso, puesto que ilustra el tipo de interacción a doble banda entre investigación sobre el desarrollo y política social que yo considero esencial para avanzar en nuestro conocimiento sobre los contextos que modelan el curso del desarrollo humano.

En concreto, sugiero que la alienación de los niños y jóvenes y sus destructivas secuelas para el desarrollo, reflejan una ruptura de las interrelaciones entre los diversos segmentos de la vida del niño: familia, escuela, grupo de iguales, vecindad y el atrayente (o, por el contrario, demasiado indiferente o recalcitrante) mundo laboral. Es, por tanto, una responsabilidad social y una oportunidad científica para el investigador, tanto de campo como experimental, que esclarezcan la naturaleza, consecuencias $y$ valor potencial de estas interrelaciones para el bienestar de los individuos y de la sociedad en su conjunto.

\section{EL IMPACTO INDIRECTO DEL TRABAJO COMO CONTEXTO PARA EL DESARROLLO HUMANO}

La referencia al mundo del trabajo hecha en el anterior punto nos anuncia el último, más distante y quizá más preñado de implicaciones, de los contextos que afectan al desarrollo del niño en la sociedad contemporánea, aun cuando sea un escenario que raramente se alcanza antes de la edad adulta. La clave del asunto puede enunciarse muy sencillamente: desde hace ya tiempo abrimos de par en par las puertas a la incorporación activa de la mujer en la vida económica. Por primera vez, el último año más de la mitad de las madres de nuestro país mayores de dieciocho años se habian incorporado a las fuerzas laborales: Pero aunque la sociedad permite y estimula esta incorporación, se han mantenido los viejos horarios del varón (de nueve a cinco) con posibles ampliaciones «alli donde las necesitemos». Paralelamente, nos hemos resistido a seguir los pasos adoptados por otros países industrializados, como el de adoptar programas a gran escala de cuidado sustitutivo para los niños de los padres trabajadores (Kamerman y Kahn, 1978). El resultado es una situación en la que muchos miles de padres estadounidenses no pueden encontrar o costearse una custodia satisfactoria para sus hijos (Toward a National Policy for Children and Families, 1976). Hemos sido también reacios a experimentar otras soluciones adoptadas ampliamente por determinados países, como un periodo de baja laboral para el cuidado del niño (Suecia) o unos horarios flexibles (Alemania).

* Nota de Tr.: Pensamos que el autor se refiere al episodio de la Biblia en que una mano misteriosa escribe ante el rey Baltasar y sus invitados las palabras que luego descifrará Daniel y que sentencian el fín de su reinado y de su reino (Dan. $\$ .1-28$ ). 
Para los estudiosos del desarrollo humano, emerge como tema crucial las consecuencias para las familias y los niños de estas nuevas circunstancias y los efectos de las posibles medidas a adoptar. Estos interrogantes no pueden ser contestados mediante la simple comparación entre madres que trabajan y madres que no lo hacen o entre niños criados en casa frente a niños que van a guarderías. Lo que se necesita es una evaluación de las fuerzas ambientales que soportan las familias de nuestra sociedad y los efectos de estas experiencias sobre las propias familias como sistemas de crianza del niño. Mis colegas y yo mismo estamos llevando a cabo ese tipo de investigación en cinco países industrializados. Cuando escribo este artículo nos encontramos en la mitad de nuestro trabajo y no poseemos aún datos sistemáticos, pero tenemos una impresión bastante firme de lo que ocurre, al menos en lo que hemos obtenido de nuestra propia muestra americana. Cualquiera que sea su nivel socioeconómico, grupo étnico o tipo de estructura familiar, no hemos encontrado aún un padre que no esté profundamente comprometido en asegurar el bienestar de su hijo. La mayoría de las familias están haciéndolo lo mejor que pueden bajo circunstancias dificiles: lo que tenemos que intentar es cambiar las circunstancias, no las familias.

\section{Notas}

1 Hayes, D., y Grether, J.: The scbool year and vacation: When do students learn? Comunicación presentada en la reunión de la Eastern Sociological Association, Nueva York, abril 1969.

2 RINGI.ER, N.: Motber's speech to ber two-year-old child: Its effect on speecb and languiage comprebension at five years. Comunicación presentada en la reunión de la Pediatric Research Society, St: Louis, abril 1977.

3 Pederson, F. A.: Motber, fatber, and infant as an interaction system. Comunicación presentada en la reunión de la American Psychological Association, Washington, D. C., septiembre, 1976.

4 Hetherington, E. M.; Cox, M., y Cox R.: Tbe devilopment of ebildren in motber-beaded families. Comunicación presentada en la Conference of Families in Contemporary America, George Washington University, Washington, D. C., junio, 1977.

5 HaRnischfeger, A., y WILEY, D. E.: Acbievement test score decline: Do we need to worry? Chicago: Central Midwest Regional Educational Laboratory, 1975.

\section{Referencias}

Belsky, J., \& Steinberg, L. D.: «The effects of day care: A critical review». Cbild Development, 1978, 49, 929-949.

Bronfenbrenner, U.: "Two worlds of childhood: U. S. and U.S.S.R.». New York: Russel Sage Foundation, 1970 .

BRONFENBRENNER, U.: «Is early intervention effective? A report on longitudinal evaluations of preschool programs» (Vol. 2). Washington, D. C.: U. S. Departament of Health, Education, and Welfare, Office of Child Development, 1974.(a).

BronfENBRENNER, U.: "The origins of alienation». Scientific American, 1974, 23I, 53-61 (b).

BRONFENBRENNER, U.: "Reality and research in the ecology of human development». Proceedings of the American Philosophical Sociey, 1975, 119, 439-469.

BRONFENBRENNER, U.: "Who needs parent education?" Teachers College Record, 1978, 79, 767-787.

BRONFENBRENNER, U.: "The ecology of human development: Experiments by nature and desing". Cambridge, Mass.: Harvard University Press, 1979.

CoChran, M. M.: «A comparison of group day and family child-rearing patterns in Sweden». Child Development, $1977,48,702-707$.

COMMITTEE ON THE JUDICIARY OF THE UNITED STATES SENATE: "Our nation's schools-A report card»: "A» in school violence and vandalism. (Informe preliminar del subcomité para investigar la delincuencia juvenil). Washington, D. C.: U. S. Government Printing Office, 1979.

Elder, G. H. JR.: «Children of the Great Depression». Chicago: University of Chicago Press, 1974.

ELDER, G. H., JR., y ROCKWELL, R. C.: "Economic depression and post-war opportunity»: A study of life patterns in hell. In R. A. Simmons (Ed.), Researcb in community and mental bealth. Greenwich, Conn.: Jai Press, 1978. 
FAnshel, D., y ShINN, E. B.: Cbildren in foster care: a longitudinal investigation. Nueva York: Columbia University Press, 1978.

Gruber, A. R.: Cbildren in foster care: Destitute, neglected, betrayed. Nueva York. Human Services Press, 1978.

HALES, D. J.; LOzOFF, B.; SOSA, R., y KENNELL, J. H.: Defining the limits of the matemal sensitive period. Developmental Medicine and Child Newrology, 1977, 19, 454-461.

Hetherington, E. M.; Cox, M., y Cox, R.: Divorced fathers. Family Coordinator, 1976, 25, 417-428.

Hetherington, E. M.; Cox M., y Cox R.: The aftermath of divorce. En J. H. Stevens, Jr., y M. Mathews (Eds.), Motber-cbild, fatber-cbild relations. Washington, D. C.: National Association for the Education of Young Children, $197^{8}$.

Kamerman, S. B., y Kahn, A. J.: Family polig: Government and families in fourteen countries. Nueva York: Columbia University Press, 1978.

Kennell, J. H.; Trause, M. A., y Klaus, M. H.: Evidence for a sensitive period in the human mother. En CIBA Foundation Symposium 33 (New series). Amsterdam: Elsevier, 1975.

LAMB, M. E.: Tbe role of the fatber in cbild development. Nueva York: Wiley, 1976.

LAzAr, I.; Hubbell, V. R.; MURRAY, H.; Rosche, M., \& RoYCE, J.: Persistence of prescbool effects: Final report (Grant No. 18-76-07843, Administration for Children, Youth, and Families). Washington, D.C.: US Department of Health, Education, and Welfare, Office of Human Development Services, 1977.

LEwn, K.: $A$ dynamic tbeory of personality. Nueva York. MacGraw Hill, ig6s.

OGBU, J. U.: The next generation: An etbnograpby of education in an urban neigbborbood. Nueva York: Academic Press, 1974.

PARKE, R. D.: Interactional design and experimental manipulation: The field lab interface. En R. B. Cairns (Ed), Social interaction: Methods, analysis, and illustration. Hillsdale, N. J.: Erlbaum, 1978.

RuOPP, R.; TRAVERS, J.; GLANTZ, F., y COEI.EN, C.: Cbildren at the center: Summary findings and their implications. (Final report of the National Day Care Study; Vol. 1). Cambridge, Mass.: Abt Associates, 1979.

SCARR-SALAPATEK, S., y WILLIAMS, M. L.: «The effects of early stimulation on low-birth-weight infants». Cbild Development, 1973, 44, 94-101.

Sherif, M.; HARveY, O. J.; HoYT, B. J.; HoOD, W. R., y SHERIF, C.: Intergroup conflict and cooperation: Tbe Robbers Cave experiment. Norman: University of Oklahoma Book Exchange, I961.

SMITH, M. B.: "School and home: Focus on achievements. En A. H. Passow (Ed.), Developing programs for the educationally disadvantaged. Nueva York: Teachers College Press, 1968.

TIzARD, B., y HODGES, J.: The effect of early institutional rearing on the development of 8-year-old children. Joxmal of Cbild Psycbology and Psycbiatry, 1978, 19, 99-1 18.

Toward a national polity for cbildren and families. Washington, D.C.: National Academy of Sciences, 1976. 